\title{
FORUM
}

\section{Europäische Parteien: Aufbruch in eine neue demokratische EU}

\author{
Jo Leinen*
}

Nach sechzig Jahren fortschreitender Integration scheint in letzter Zeit zunehmend Sand ins Getriebe der Europäischen Union zu kommen. Immer häufiger wird die Kluft zwischen ,Brüssel ${ }^{`}$ und seinen Institutionen sowie den Mitgliedstaaten und ihren Bürgerinnen und Bürgern angeprangert. Der Mehrheit der Unionsbürger ist nicht klar, was und wie auf europäischer Ebene entschieden wird; sie fordern, stärker in die Entscheidungen der EU eingebunden zu werden. ${ }^{1}$ Einer der Hauptgründe für dieses Defizit ist die fehlende Verbindung zwischen den europäischen Institutionen und den Unionsbürgern sowie zwischen den Wählern und den Abgeordneten. Während diese Funktion auf nationaler Ebene zum großen Teil von den Parteien übernommen wird, ist die Existenz Europäischer Parteien als Bindeglied zwischen Unionsbürgern und Unionspolitik nur gering ausgeprägt.

Erst in den letzten Jahren haben sich Europäische Parteien herausgebildet und begonnen, ihre Funktion als Transmissionsriemen zwischen Bürgern und Politikern wahrzunehmen. 1992 gelang es mit der Unterzeichnung des Maastrichter Vertrages eine Rechtsgrundlage für Europäische Parteien im Primärrecht der EU zu verankern. In Artikel 138a des Vertrages (heute Artikel 191 EGV) heißt es: „Politische Parteien auf europäischer Ebene sind wichtig als Faktor der Integration in der Union. Sie tragen dazu bei, ein europäisches Bewusstsein herauszubilden und den politischen Willen der Bürger der Union zum Ausdruck zu bringen." Trotz dieser Rechtsgrundlage gelang es erst im Jahre 2003 durch Verabschiedung der „Verordnung über die politischen Parteien auf europäischer Ebene und deren Finanzierung “2 die finanzielle Eigenständigkeit Europäischer Parteien und ihre Unabhängigkeit von den Fraktionen im Europäischen Parlament sicherzustellen, wie es schon lange Zeit vom Europäischen Gerichtshof eingefordert wurde. Dies wurde nur möglich, nachdem zuvor mit dem Nizza-Vertrag die Regelung eingeführt wurde, in dieser Frage mit qualifizierter Mehrheit abzustimmen.

In diesem Beitrag wird der Mehrwert Europäischer Parteien für die europäische Demokratie dargestellt. Die verschiedenen Herausforderungen für Europäische Parteien werden erläutert, anschließend werden notwendige weitere Schritte zur Stärkung Europäischer Parteien skizziert.

* Jo Leinen, MdEP, Vorsitzender des Ausschusses für Konstitutionelle Angelegenheiten des Europäischen Parlaments, Berichterstatter zum Statut für Europäische Parteien.

Der Beitrag entstand unter der Mitarbeit von Jan Kreutz und Philipp Hessel.

1 Laut Eurobarometer geben 78 Prozent der Befragten an, die EU-Institutionen sollten Anstrengungen unternehmen, um die Bürger besser in die Angelegenheiten der Europäischen Union einzubeziehen. Vgl. Europäische Kommission, Eurobarometer, EB 63 Frühjahr 2005, S. 37-41, abrufbar unter: http://europa.eu.int/comm/ public_opinion/archives/eb/eb63/eb63.4_de_first.pdf (letzter Zugriff: 7/19/06).

2 Verordnung (EG) Nr. 2004/2003 des Europäischen Parlaments und des Rates vom 4. November 2003 über die Regelungen für die politischen Parteien auf europäischer Ebene und ihre Finanzierung. 


\section{Europäische Parteien sind unverzichtbar für die europäische Demokratie}

Europäische Demokratie ist nicht denkbar ohne starke Europäische Parteien. Abgesehen von den europäischen Institutionen (insbesondere dem Europäischen Parlament und der Europäischen Kommission) sowie einigen pro-europäischen Nichtregierungsorganisationen gibt es kaum Akteure, die Bürger über europäische Themen informieren und europaweite Debatten führen. Im Europäischen Rat und im Ministerrat üben die nationalen Regierungen zwar großen Einfluss auf EU-Entscheidungen aus. Sie nehmen jedoch in erster Linie die jeweiligen nationalen Interessen wahr und vertreten nur selten die in Brüssel getroffenen europäischen Beschlüsse auch zu Hause gegenüber der Öffentlichkeit. ,Brüssel ‘ wird zum Sündenbock für unangenehme Entscheidungen gemacht. Diese Negativkommunikation über Europa hat zur Distanz zwischen Bürgern und den europäischen Institutionen beigetragen.

Dass die Einführung des Euros eine gute Sache für Europa und die europäische Wirtschaft ist, wurde in Deutschland kaum diskutiert, wohl aber die Symbolkraft der D-Mark. Dass die Erweiterung bereits heute sehr positive Folgen hat, wird in der öffentlichen Debatte kaum thematisiert, wohl aber, dass einige Regionen vorübergehende Probleme durch die Erweiterung haben. Die EU wird von der politischen Klasse in den Mitgliedstaaten in erster Linie aus nationaler Perspektive beleuchtet, der mögliche Mehrwert für die gesamteuropäische Entwicklung wird dabei selten berücksichtigt. Wenn die EU sich zu einer politischen Union weiterentwickeln soll, in der es um mehr als einen gemeinsamen Binnenmarkt geht, dann ist eine in allen Mitgliedstaaten und darüber hinaus auf europäischer Ebene geführte politische Debatte über die Zukunft der EU unverzichtbar.

Eine solche Debatte ist in einem hohen Maß auf Europäische Parteien angewiesen. Konservativen, Sozialdemokraten, Grünen, Liberalen und anderen politischen Familien muss es gelingen, Themen transnational zu debattieren und Positionen zu formulieren, die für alle nationalen Gliederungen verbindlich sind. Die Unionsbürger müssen in die Lage versetzt werden, sich bei den Europawahlen für eine Partei zu entscheiden, die über ein konkretes politisches Programm verfügt und die eine überzeugende Führungspersönlichkeit nominiert. Europapolitik würde so Profile und Gesichter bekommen, die Politik der EU würde bürgernäher und eine größere Beteiligung bei den Europawahlen könnte erreicht werden.

Im Gegensatz zu föderalen Strukturen wie in der Bundesrepublik Deutschland fehlt in der Europäischen Union der Kitt zwischen nationaler und europäischer Ebene. In der Bundesrepublik wird durch die Zugehörigkeit der verschiedenen Ministerpräsidenten, Parlamentarier und Bundesminister zu den Parteien gewährleistet, dass die Landesebene politisch mit der Bundesebene verzahnt ist. Auf europäischer Ebene gibt es noch keine Partei, die gewährleisten kann, dass europäisch definierte Interessen über die Interessen eines Nationalstaats gestellt werden. Während sich bei den Fraktionen im Europäischen Parlament bereits eine europäische Programmatik entwickelt, überwiegen im Ministerrat auf der Ebene der Regierungen nach wie vor die nationalen Interessen. Eine gemeinsame Linie der Parteien ist dort bisher nur selten zu beobachten.

\section{Hindernisse auf dem Weg zu starken Europäischen Parteien}

Bis Europäische Parteien eine ähnliche, integrierende Rolle spielen können wie in den Mitgliedstaaten müssen eine Reihe von Hindernissen überwunden werden. Auch wenn durch den Leinen-Bericht zur Finanzierung von Europäischen Parteien ${ }^{3}$ wichtige Grundla-

3 Verordnung (EG) Nr. 2004/2003 des Europäischen Parlaments und des Rates über die Regelungen für die politischen Parteien auf europäischer Ebene und ihre Finanzierung vom 4. November 2003 und Entschließung des Europäischen Parlaments zu Europäischen Politischen Parteien (2005/2224 (INI)) vom 23. März 2006. 
gen für eine bessere Arbeit der grenzüberschreitenden Parteienzusammenschlüsse geschaffen wurden, sind noch eine Reihe von Herausforderungen zu bestehen.

\section{Die parteien-unfreundliche Struktur der EU}

Auf nationaler Ebene haben Parteien klare Funktionen: die Erarbeitung eines politischen Programms, das für die zukünftige Regierungsarbeit maßgeblich sein sollte; die Ausbildung und Bereitstellung des politischen Führungspersonals und die Rückkopplung von Entscheidungen der Parlamente und Regierungen zu den Wählern. Diese Funktionen wurden in der EU bisher nicht oder nur von anderen Institutionen wahrgenommen.

Ein ,richtiges ‘ politisches Programm für die EU ist kaum erkennbar. Die Zielrichtung der EU wird größtenteils durch Einzelentscheidungen in den verschiedenen Politikfeldern vorgegeben. Wenn eine neue Kommission ihre Arbeit aufnimmt, bleibt sie weiterhin an die Vorlagen des Vorgängerkollegiums gebunden. Das politische Programm folgt Entscheidungen des Tagesgeschäfts, nicht andersherum. Bei legislativen Entscheidungen auf Ebene des Rates sind politische Richtungen darüber hinaus nachrangig. Die nationalen Administrationen und Regierungen folgen ihren national definierten Präferenzen, nicht aber den Interessen der Parteienfamilie. Dies hat zur Folge, dass es für Europäische Parteien bisher schwierig ist, sich politisch zu profilieren: Warum sollte der Bürger bei der Europawahl für eine Partei stimmen, deren politische Position nicht erkennbar und noch dazu offenbar irrelevant ist? Erschwerend kommt hinzu, dass der Europawahlkampf in den Mitgliedstaaten kaum auf europäische Themen ausgerichtet ist. Aufgrund der geringen Medienaufmerksamkeit bei europapolitischen Debatten erscheinen diese Wahlen vielen Bürgern als nicht wichtig. Unter diesen Bedingungen fällt es den Europäischen Parteien schwer, sich attraktiv zu präsentieren. Es kommt hinzu, dass aus der derzeitigen Rechtslage der Verträge nicht klar hervorgeht, ob Europäische Parteien überhaupt Wahlkampagnen durchführen dürfen.

Auch auf die Auswahl der zukünftigen Führungspersönlichkeiten haben die Europäischen Parteien kaum Einfluss. Der Vorschlag für das Amt des Kommissionspräsidenten wird bisher von den Mitgliedstaaten hinter verschlossenen Türen verhandelt. Seitdem die Wahl des Kommissionspräsidenten den Wahlen zum Europa-Parlament folgt steigt jedoch die Chance, dass die für diese Position gefundene Person der Parteienfamilie angehört, die die Europawahlen gewonnen hat. Auf den Vorschlag nehmen aber nicht in erster Linie die Gremien der Europäischen Parteien Einfluss, sondern die Regierungschefs der Mitgliedstaaten. Kommissionspräsidenten, die zuvor Premierminister oder Kabinettsmitglieder waren, haben Erfahrungen in Ministerräten oder Regierungskonferenzen gesammelt, nicht aber in den Leitungsgremien einer Europäischen Partei. Es ist daher anzunehmen, dass die von ihnen zu erwartende Loyalität eher auf den Ministerrat als auf die Europäische Partei ausgerichtet ist.

Die Europäischen Parteien sind derzeit kaum in der Lage, Entscheidungen der europäischen Ebene und die Meinungen der Bürger zu vernetzen. Im Gegensatz zur nationalen Ebene bestehen die Europäischen Parteien nicht aus individuellen Mitgliedern. In der Satzung der Europäischen Sozialistischen Partei wird die Parteimitgliedschaft auf die Mitglieder der nationalen Parteien beschränkt. Die Satzung der Europäischen Volkspartei (EVP) sieht zwar die Möglichkeit individueller Mitglieder vor. Genauso wie bei den Grünen und den Liberalen machen sie aber nur einen geringen Anteil der Gesamtmitgliedschaft aus. Die Europäischen Parteien sind daher nach wie vor darauf angewiesen, dass die Kommunikation über europäische Politik durch die nationalen Strukturen der Mitgliedsparteien übernommen wird. 
In Orts- und Kreisverbänden der nationalen Parteien spielt Europapolitik bisher kaum eine Rolle. Von den Parteimitgliedern wird die Diskussion europäischer Themen als nicht notwendig erachtet, die Entscheidungen scheinen fern von der politischen Realität der Arbeit vor Ort. Wenn die Europäischen Parteien ihre Rolle als Bindeglied zwischen europäischen Entscheidungen und Bürgerinteressen wahrnehmen wollen, muss es ihnen gelingen, auch die lokale Ebene zu ereichen. Um dies zu gewährleisten, hat zum Beispiel die Sozialdemokratische Partei Europas (SPE) kürzlich ein Netzwerk von ,PES activists ' gegründet. Mitglieder der nationalen Parteien können sich online registrieren und haben so die Möglichkeit, über europäische Themen ihres Interesses auf einer speziellen Homepage zu diskutieren und an gemeinsamen Aktivitäten, wie beispielsweise einer Sommeruniversität, teilzunehmen. Dies scheint ein erster wichtiger Schritt zur notwendigen Stärkung der Mittlerposition der Europäischen Parteien zwischen Bürgern und politischer Führung. Auch mit Blick auf die Parteiführungen der nationalen Parteien und die Abgeordneten ist eine Europäisierung im Sinne einer Perspektiverweiterung inhaltlich und strukturell voranzutreiben.

Es wird kaum möglich sein, die Strukturen der nationalen Parteien Eins zu Eins auf die europäische Ebene zu übertragen. Damit Europäische Parteien ihrer wichtigen Rolle für die Demokratie in der EU gerecht werden können, müssen sie sich jedoch weiterentwickeln und starke Akteure werden. Die Europäische Verfassung sieht beispielsweise vor, dass das Europäische Parlament den Kommissionspräsidenten zukünftig auf Grundlage der Wahlergebnisse wählt. Dies muss zur Folge haben, dass die Parteien mit einem gemeinsamen Spitzenkandidaten bei den Europawahlen antreten. Die Partei mit den meisten Stimmen bekommt so die Möglichkeit, ihren Spitzenkandidaten für das Amt des Kommissionspräsidenten durchzusetzen. Die Wähler hätten somit eine direkte Entscheidungsmöglichkeit über das Führungspersonal der nächsten Legislaturperiode sowie das ,Regierungsprogramm‘. Um diese in der Verfassung vorgesehene wichtige Änderung umzusetzen, müssen die Europäischen Parteien in der Lage sein, eine(n) gemeinsame(n) Kandidaten/in und ein gemeinsames Programm aufzustellen.

\section{Die Schwierigkeiten einer gemeinsamen Politik}

Neben diesen strukturellen Problem besteht die Aufgabe für die Europäischen Parteien vor allem darin, gemeinsame Positionen zu entwickeln und zu vertreten. Die nationalen Profile der Parteien haben sich über hundert Jahre unabhängig voneinander entwickelt. Zwar gab es Politiker wie Willy Brandt, die im Exil auch in anderen Parteien Erfahrungen gesammelt haben. Im Wesentlichen haben aber nationale Strukturen und die Wünsche der nationalen Wähler die Führungspersönlichkeiten der Parteien geprägt. So ist es nicht verwunderlich, dass die Parteien in Europa verschiedenen Traditionen und Kulturen folgen.

Am größten sind diese Unterschiede wohl bei der EVP, die sich aus verschiedenen konservativen, christdemokratischen, Zentrums- und Bauernparteien zusammensetzt. So sind die Differenzen zwischen britischen Konservativen und deutschen Christdemokraten zu Fragen der europäischen Integration sehr groß.

Doch auch in der SPE ist die interne Positionsbestimmung zu politischen Themen schwierig. Zwar gibt es die Sozialistische Internationale SI (Socialist International), die es den nationalen Parteien schon vor Gründung der SPE ermöglichte, in regem Austausch zu stehen. Dies hat aber noch nicht dazu geführt, dass sich einheitliche Positionen in wichtigen Politikfeldern herausbilden konnten. Weder über die Frage der Europäischen Verfassung oder der Zukunft des europäischen Sozialmodells, noch über die Frage der europäischen Sicherheitspolitik lässt sich ein Konsens erzielen. Bei letzterem stehen sich beispielsweise antimilitaristische Positionen und pragmatischere Überlegungen gegenüber. 
Bei den liberalen Parteien gibt es ebenfalls große Unterschiede in der Gewichtung von sozialliberalen oder wirtschaftsliberalen Positionen. Auch wenn die Grüne Partei jünger ist, haben sich bei ihr sehr verschiedene inhaltliche Positionierungen herausgebildet, wie die Abspaltung der anti-europäischen nordischen Grünen zeigt. Die anderen Europäischen Parteien haben eine noch sehr junge Geschichte, sodass eine Beurteilung ihrer Positionierung bisher schwer fällt.

Bei allen Parteien ist es äußerst kompliziert, gemeinsame Positionen festzulegen. Dies zeigt sich bei den Wahlmanifesten, die oftmals sehr vage sind und wenig konkrete politische Versprechen für den Fall eines Wahlsieges enthalten. Der Bürger kann unter diesen Umständen nur schwer erahnen, welche Partei nach der Wahl welche Maßnahmen ergreifen wird. Eine geringe Wahlbeteiligung ist angesichts dieser Situation nicht verwunderlich.

Es sind aber in jüngster Zeit Veränderungen festzustellen. So haben insbesondere die Mitglieder der SPE, nicht nur die Fraktion im Parlament, sondern auch Minister und Regierungschefs, bei den Verhandlungen zur Dienstleistungsrichtlinie eine gemeinsame Position vertreten und so entscheidend zu einer Abänderung des ursprünglichen Richtlinienentwurfs beigetragen. Auch im Verfassungskonvent hat sich gezeigt, dass die Parteien die Notwendigkeit eines gemeinsamen Auftretens erkannt haben. In der Schlussphase des Konvents wurden die letzten offenen Entscheidungen vor allem innerhalb und zwischen den Parteienfamilien getroffen.

\section{Die Herausforderung eines gemeinsamen Kandidaten}

Wenn die Europäischen Parteien eine entscheidende Rolle bei der Auswahl der politischen Führungskräfte der Union spielen sollen, müssen sie in der Lage sein, gemeinsame Kandidaten aufzustellen. Schon heute wäre es möglich, dass die Europäischen Parteien Spitzenkandidaten für die Wahl des Präsidenten der Europäischen Kommission aufstellen. Diejenige Partei, die die Wahlen des EP gewinnt, könnte dann mit Unterstützung der Regierungschefs ihrer Partei diese(n) Kandidaten/in vorschlagen. So hat die EVP vor den letzten Europawahlen im Juni 2004 deutlich gemacht, dass sie im Falle eines Wahlsieges Anspruch auf das Amt des Kommissionspräsidenten stellt. Zwar hatte der EVP-Wunschkandidat JeanClaude Juncker aus Luxemburg abgewinkt. Guy Verhofstadt aus Belgien bekundete Interesse an dieser Aufgabe, setzte sich aber nicht durch, da er den Liberalen angehört. Letztlich einigte sich die EVP auf den portugiesischen Premierminister José Manuel Barroso von der liberal-konservativen Partido Social Democrata (PSD), die Mitglied der EVP ist.

Im Gegensatz zu den nationalen Parteien, bei denen die Aufstellung eines Kandidaten für das Amt des Regierungschefs eine der wichtigsten Aufgaben darstellt, gibt es auf europäischer Ebene damit noch wenig Erfahrung. Nationale Parteien, die eine Regierung in einem Mitgliedstaat stellen, akzeptierten bisher nicht die Nominierung eines Kandidaten für den Kommissionspräsidenten durch Mehrheitsentscheidung innerhalb der Europäischen Partei. Da sie später bei der Sitzung des Europäischen Rates die Möglichkeit hatten, jeden unliebsamen Kandidaten durch ein Veto zu blockieren, konnte eine parteiinterne Nominierung demnach bisher nur einstimmig erfolgen. Dies ist jedoch bei Mitgliedsparteien aus bis zu 25 Staaten ein äußerst schwieriges Unterfangen. Zwar hatte die Grüne Partei bei der letzten Wahl des Europäischen Parlaments Daniel Cohn-Bendit als Spitzenkandidat nominiert. Dies war auch deshalb etwas leichter, weil die grüne Partei keine Aussichten hatte, die stärkste Partei zu werden. 


\section{Die Zukunft Europäischer Parteien}

Bis die Europäischen Parteien eine zentrale Rolle in der Politikgestaltung der EU spielen und den Nukleus einer europäischen Demokratie bilden wird noch Zeit vergehen. Es wurden in den letzten Jahren aber viele wichtige Schritte in die richtige Richtung getan.

\section{Verfassungsordnung der EU}

Eine erste Voraussetzung für stärkere Europäische Parteien ist die zunehmende Konstitutionalisierung der Europäischen Union. Die in der Europäischen Verfassung vorgesehenen institutionellen Reformen für die EU weisen in die richtige Richtung. Eine sehr wichtige Entwicklung ist die Stärkung des Europäischen Parlaments durch die Einführung der Mitentscheidung in vielen weiteren Politikfeldern. Dadurch wird die Möglichkeit der Parteien, die politische Richtung der EU zu bestimmen, verbessert. Insbesondere der verstärkte Einfluss des Parlaments auf das Budget der EU wird den Parteien erlauben, eine größere Rolle bei der politischen Prioritätensetzung der EU zu spielen. Auch die Änderung bezüglich der Wahl des Kommissionspräsidenten wird den Europäischen Parteien erlauben, bei der Auswahl der politischen Führung der EU eine wichtige Rolle zu spielen. Durch die in der Verfassung vorgesehenen Änderungen der Institutionen nähert sich die EU dem demokratischen Ziel an: mit seiner Stimme kann der Bürger, mit Hilfe der Parteien als Bindeglieder, auf die politische Ausrichtung und das politische Führungspersonal der EU Einfluss nehmen.

\section{Ein europäisches Parteienstatut}

Neben der Verfassung bedarf es auch spezifischer Bestimmungen für die Arbeitsweise und Funktionsfähigkeit der Europäischen Parteien. Dies sollte in einem europäischen Parteienstatut festgelegt werden. Am 23. März 2006 hat das Europäische Parlament eine Entschließung angenommen, in der die Europäische Kommission aufgefordert wird, einen Entwurf für solch ein Parteienstatut dem Parlament und dem Rat zur Entscheidung vorzulegen. ${ }^{4}$ Darin sollen Einzelheiten über die Funktionsweise, Aufgaben und Finanzierung von Europäischen Parteien geregelt werden. Bis heute sind die Europäischen Parteien als Vereine nach nationalem Recht registriert, meist in Belgien. Ziel für ein Statut ist es, dass die Parteienfamilien eine europäische Rechtspersönlichkeit erhalten, durch die sie in allen Mitgliedstaaten der EU über dieselben Rechte und Pflichten verfügen. Ferner muss in dem Statut geklärt werden, inwiefern die individuelle Mitgliedschaft in den Parteien ermöglicht wird. Auch Regeln für die innere Demokratie in den Parteien, die Aufstellung von Kandidaten sowie die Willensbildung bei Parteikongressen sollten in solch einem Statut verankert werden.

Auch wenn die Teilnahme von Europäischen Parteien an europäischen Wahlkämpfen, Referenden und Kampagnen als selbstverständlich erscheint, ist dies in der EU-Gesetzgebung bisher nicht eindeutig vorgesehen. Dahinter steckt die Angst, die Europäischen Parteien könnten auf nationale Wahlkämpfe einwirken. Ein europaweiter politischer Diskurs kann aber nur entstehen, wenn zu inhaltlichen Problemen von den Europäischen Parteien konkurrierende Lösungen angeboten werden können. Dies muss mit dem Europäischen Parteienstatut ermöglicht werden.

4 Entschließung des Europäischen Parlaments zu Europäischen Politischen Parteien (2005/2224 (INI)) vom 23. März 2006. 


\section{Europäische politische Stiftungen}

Insbesondere die Erfahrung in Deutschland hat gezeigt, dass neben den politischen Parteien auch politische Stiftungen eine wichtige Rolle zu erfüllen haben. Sie fördern die politische Bildung in der Gesellschaft, mit Hilfe von Studienförderungen helfen sie bei der Ausbildung zukünftiger politischer Führungskräfte, sie unterstützen den Aufbau demokratischer Parteiensysteme weltweit und dienen nicht zuletzt als Think Tanks für die Parteien. Entscheidend ist, dass politische Stiftungen zwar unabhängig von Parteiinteressen agieren, aber andererseits durch ihre Nähe zu den ideologischen Grundausrichtungen der Parteien den Bürgern Wahlmöglichkeiten zwischen verschiedenen Alternativen geben. Auch auf europäischer Ebene besteht eine große Notwendigkeit für solche Aktivitäten. Dies wird sowohl im Weißbuch Kommunikation der Europäischen Kommission als auch im Leinen-Bericht des Europäischen Parlaments zu Europäischen Parteien unterstrichen. Wie die Diskussionen zur europäischen Verfassung gezeigt haben, stecken europaweite politische Debatten in den Kinderschuhen. Europäische politische Stiftungen können einen wichtigen Beitrag leisten, indem sie transnationale Diskurse organisieren. Auch europäische Stipendienprogramme könnten einen wichtigen Beitrag leisten, indem sie bei jungen Führungskräften die europäische Dimension in allen gesellschaftlichen Bereichen verdeutlichen. Die Gründung europäischer politischer Stiftungen und ihre finanzielle Absicherung wäre daher ein weiterer Schritt zur Stärkung der europäischen Demokratie.

\section{Politische Jugendorganisationen}

Auch die Jugendorganisationen der Parteien müssen gestärkt werden. Sie spielen eine wichtige Rolle bei der Ausbildung zukünftiger europäischer Führungspersönlichkeiten der Parteien. Die heute schon existierenden Parteijugendorganisationen organisieren vielfältige Veranstaltungen auf europäischer Ebene, wodurch sichergestellt wird, dass auch die zukünftigen Politiker der nationalen Ebene frühzeitig an europäische Themen herangeführt werden. In einem Europäischen Parteienstatut oder einem gesonderten Gesetz muss daher sichergestellt werden, dass europäische Jugendorganisationen anerkannt werden und ausreichend finanzielle Ressourcen zur Verfügung gestellt bekommen.

\section{Ausblick}

Die Europäische Verfassung hat das Ziel der partizipativen und der parlamentarischen Demokratie in der EU vorgegeben. Nur eine demokratische EU wird eine Bürgerunion sein und Vertrauen wie auch Unterstützung durch die Menschen erhalten. Europäische Parteien sind ein unverzichtbares Instrument zur Realisierung der Europäischen Demokratie. Bis zu den Europawahlen im Juni 2009 müssen erkennbare Fortschritte erzielt werden, sowohl für das politische Programm, als auch für das den Bürgern vorgeschlagene europäische Führungspersonal. 